\title{
COMMISSIONING RESULTS OF ANKA
}

\author{
D.Einfeld ${ }^{\S}$, E.Huttel ${ }^{\S}$, F.Perez, M.Pont and G.K.Sahoo, \\ ANKA GmbH, Hermann-von-Helmholtz-Platz 1, D-76344 Leopoldshafen, Germany \\ ${ }^{\$}$ FGS, Forschungszentrum Karlsruhe, P.O. 3640, D-76021 Karlsruhe, Germany \\ $\S$ on leave from INDUS, Indore, India
}

\begin{abstract}
ANKA is a $2.5 \mathrm{GeV}$ synchrotron radiation facility located at Forschungszentrum Karlsruhe (Germany). ANKA consists of a $500 \mathrm{MeV}$ injector and a $2.5 \mathrm{GeV}$ storage ring. The commissioning of the storage ring started in December 1999. A $100 \mathrm{~mA}$ electron beam at $2.5 \mathrm{GeV}$ with a minimum lifetime of 4 hours was reached in July 2000. After introducing a gap filling pattern and optimising the temperature of the RF cavities the current at $500 \mathrm{MeV}$ has reached more than $200 \mathrm{~mA}$, and $180 \mathrm{~mA}$ at $2.5 \mathrm{GeV}$. At the same time the lifetime has increased up to 10 hours. Basic beam parameters like emittance, chromaticity, and machine functions among others have been measured and found to agree with design calculations. This paper describes the status of the synchrotron radiation facility 1.5 years after starting commissioning. The beamlines are presently under commissioning, and the first experiments are under way.
\end{abstract}

\section{INTRODUCTION}

The synchrotron radiation source ANKA consists of a $500 \mathrm{MeV}$ injector [1] and a $2.5 \mathrm{GeV}$ storage ring [2]. The storage ring has a four-fold symmetry, with eight double bending magnets, four long $(5.6 \mathrm{~m})$ straight sections for insertion devices and four short straight sections $(2.2 \mathrm{~m})$. Of these one houses the injection and two others the RF cavities. The main parameters of the storage ring are given in table 1.

Table 1: Nominal parameters of ANKA at $2.5 \mathrm{GeV}$

\begin{tabular}{|l|c|c|}
\hline Parameter & Value & Unit \\
\hline Energy & $0.5-2.5$ & $\mathrm{GeV}$ \\
\hline Circumference & 110.4 & $\mathrm{~m}$ \\
\hline Momentum compaction factor & 0.008 & \\
\hline Working point $\left(\mathrm{Qx}_{\mathrm{x}}, \mathrm{Qy}\right)$ & $6.85,3.22$ & \\
\hline Natural Chromaticity $\left(\xi_{\mathrm{x}}, \xi_{\mathrm{y}}\right)$ & $-14,-9$ & \\
\hline Energy spread & 0.001 & \\
\hline Emittance & 91 & $\mathrm{nmrad}$ \\
\hline Photon critical energy & 6 & $\mathrm{keV}$ \\
\hline
\end{tabular}

\section{COMMISSIONING of the STORAGE RING}

Commissioning of the storage ring started on $7^{\text {th }}$ of December 1999. The year 2000 was dedicated to the commissioning of the accelerator itself, while the year 2001 has been mainly used to deliver synchrotron light to the beam lines and the experimental stations for their commissioning.

\subsection{Injection into the Storage Ring}

The beam extracted from the Booster is transferred to the storage ring via a $20 \mathrm{~m}$ long transfer line. The beam is horizontally injected into the storage ring from the inner side with the help of the septum magnet. Three kicker magnets create a closed orbit bump to bring the stored beam close to the septum sheet. The bump is extended over two achromats, in this way the strength of the kickers is reduced considerably but the quadrupoles have a nonnegligible influence during injection.

The efficiency of the injection process is around $25 \%$, well below the expected value. Even though the extraction line has two bending magnets and 9 quadrupoles to take care of the matching between the booster and the storage ring, we have not yet succeed to obtain a good matching during injection. We believe that the reason is that our theoretical model does not agree with the real machine. We are investigating the causes of that disagreement.

\subsection{Accumulation at $500 \mathrm{MeV}$}

The injection at ANKA is done at $500 \mathrm{MeV}$. At that energy we accumulate current until the desired value, which is afterwards ramped up to $2.5 \mathrm{GeV}$.

The main difficulties we have encountered at ANKA are related to the accumulation at $500 \mathrm{MeV}$. The beam has, at that energy, a damping time of hundreds of milliseconds, which makes the beam very sensitive to any kind of instability.

Different kind of oscillations appeared that prevented us to reach more than $100 \mathrm{~mA}$. We are investigating the source of the instabilities but preliminary results indicate that we could face the so call Fast Beam Ion Instability [3] that could shift our tune and destroy in some cases the accumulated beam. 
After improving the temperature regulation of the cavities, we overcame this problem by using the fine temperature tune of the cavities to excite the beam longitudinally with one of the longitudinal HOMs of one cavity. The complication comes because by increasing the accumulated current, the HOMs excitation become very strong and could as well bring the beam to an unstable situation. So, during accumulation we are shifting the temperature of this cavity as a function of the current in such a way that the longitudinal excitation stays in a reasonable value.

With this scheme we have been able to accumulate more than $200 \mathrm{~mA}$.

\subsection{Ramping}

Once the accumulation process finishes, the beam is ramped up to $2.5 \mathrm{GeV}$.

Dipoles, quadrupoles, sextupoles, and correctors are ramped synchronously but the RF voltage is ramped freely. The ramping is performed in two steps, first up to $1.65 \mathrm{GeV}$ and then up to $2.5 \mathrm{GeV}$. The step is mainly used to ensure that the needed RF voltage is there before reaching the maximum energy, but also it is used to allow the temperature of the cavities to stabilise. The ramping takes approximately 5 minutes.

Routinely we are ramping $150-180 \mathrm{~mA}$ up to $2.5 \mathrm{GeV}$. Attempts have been made to ramp $200 \mathrm{~mA}$ but in all the cases a vacuum trip in the cavities has prevented us to succeed.

\section{MEASUREMENTS at $2.5 \mathrm{GeV}$}

\subsection{Tunes}

The tunes are measured by exciting the beam with a spectrum analyser with a tracking generator plus amplifier through a stripline and picking up the beam response in a dedicated BPM.

The working diagram during ramping is shown in figure 1 . The tune has been adjusted to avoid crossing any resonance.

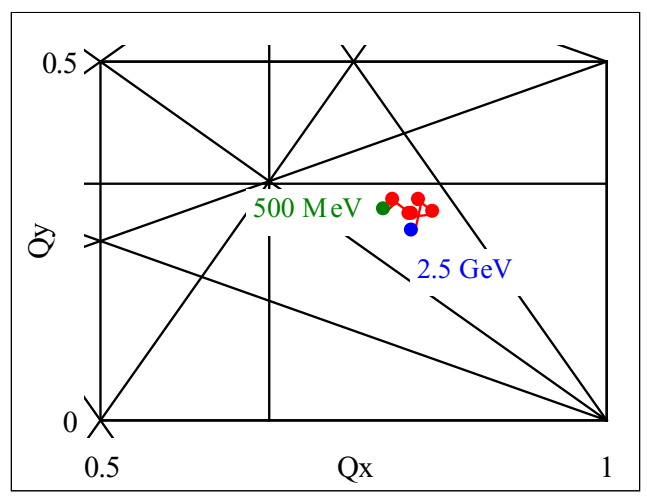

Figure 1: Working diagram during ramping, fractional tune
At $2.5 \mathrm{GeV}$, instead of working at the stable working point $(6.81,3.27)$, to increase the lifetime and to allow a more efficient vacuum cleaning by the synchrotron light we approach the sum resonance, working point $(6.83,3.19)$, which excites the beam vertically.

\subsection{Chromaticity}

The chromaticity has been obtained by measuring the tune shift at different RF frequencies, i.e. at different energy deviations, see figure 2 .

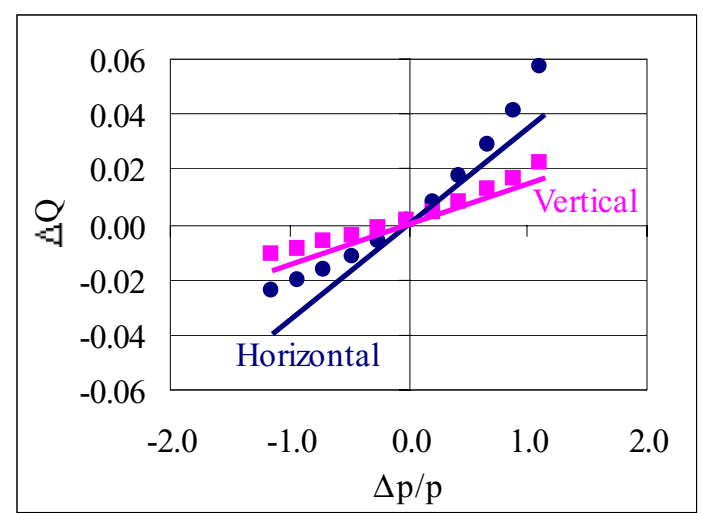

Figure 2: Horizontal and vertical tune shift vs energy deviation. The slope at zero defines the chromaticity

The obtained chromaticities are $\xi_{\mathrm{x}}=+0.04$ and $\xi_{y}=+0.02$. They are only slightly positive, but no problems have been observed with the stored beam.

\subsection{Linear Optics}

Figure 3 shows the machine functions along a cell of the storage ring in comparison with the measured ones.

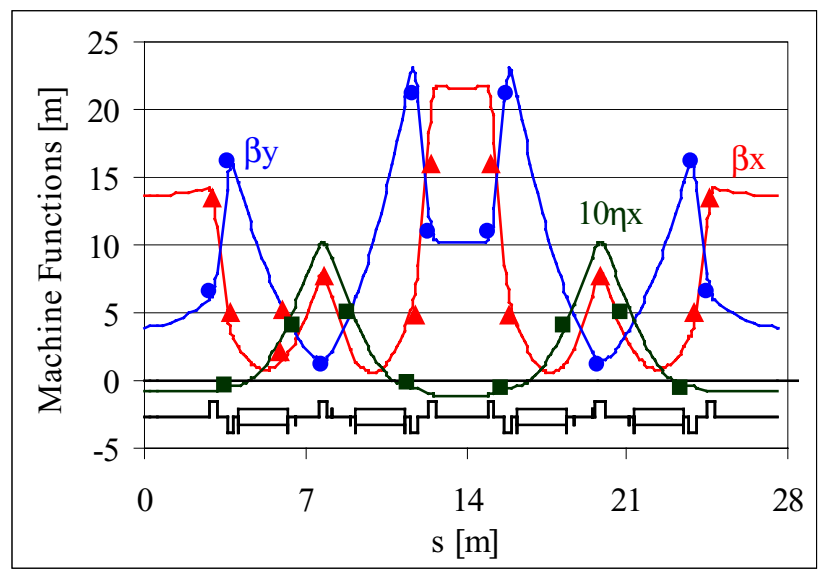

Figure 3. Machine functions.

Line: calculated; Points: Measured

The dispersion has been measured by changing the RF frequency and recording the closed orbit. The beta functions have been calculated from the tune shift due to the change in $\mathrm{k}$ of each quadrupole family.

Even though we can fit a theoretical model to reproduce the measured values, the model uses $\mathrm{k}$ values for the 
quadrupoles that are not in agreement with the ones actually applied. Work on the calibration of the settings have been done but does not explain the discrepancy. We are now investigating possible problems in the stability of one of the quadrupole power supply as a possible reason for it, but we have not yet arrived to any conclusion.

\subsection{Closed orbit correction}

Closed orbit correction relies on 32 BPM stations, 28 horizontal correctors and 16 vertical correctors. The maximum kick available is $\pm 1 \mathrm{mrad}$ in both directions.

The software for the closed orbit correction uses either a calculated or a measured response matrix for setting the correctors. The system is working properly to reduce the orbit distortion down to $1 \mathrm{~mm}$ peak to peak, but this is the actual limit. See figure 4.

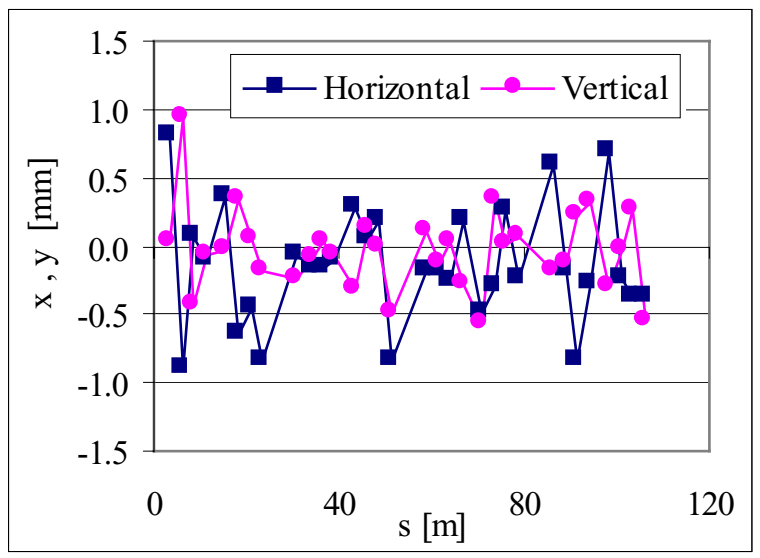

Figure 4: Closed orbit as measured by BPM's

Two reasons could be the cause of this still not optimum performance of the orbit correction. First our model of the machine is still not good enough (as explained in the previous paragraph), and second, the BPMs are not yet properly calibrated.

We have now installed the hardware needed to perform a Beam Based Alignment calibration of the BPMs but still has not been systematically used for lack of time.

We expect that after the calibration of the BPMs with the beam and a better machine model the orbit correction scheme could be improved.

\subsection{Emittance and coupling}

We measured the beam size by using one of the beam lines now in commissioning, the so called fluo-topo beam line, which allows to move two slits, in the horizontal and the vertical direction, and to record the intensity of the synchrotron light passing through a pinhole and to measure it in a photodiode.

With this method we measured the beam size in the middle of a bending magnet:

$$
\sigma \mathrm{x}=309 \mu \mathrm{m} \text { and } \sigma_{\mathrm{y}}=51 \mu \mathrm{m} \text {. }
$$

Taking the fitting to the measured machine functions at the centre of the bending, the emittance and the coupling have been calculated:

$$
\varepsilon=106 \mathrm{nmrad} \text { and } \chi=0.3 \%
$$

The emittance agrees with the results of the fitting. The coupling is better than expected.

\subsection{Lifetime}

A typical injection and storage beam is shown in figure 5. That day we injected and ramped $160 \mathrm{~mA}$ at 14:00, with a lifetime of around 10 hours. Next day at 8:00 still remained $52 \mathrm{~mA}$, which we killed to inject again.

To have this relatively large lifetime we operate in a working point close to a sum resonance to increase the beam size vertically. If we do not do that the lifetime decreases approximately $50 \%$.

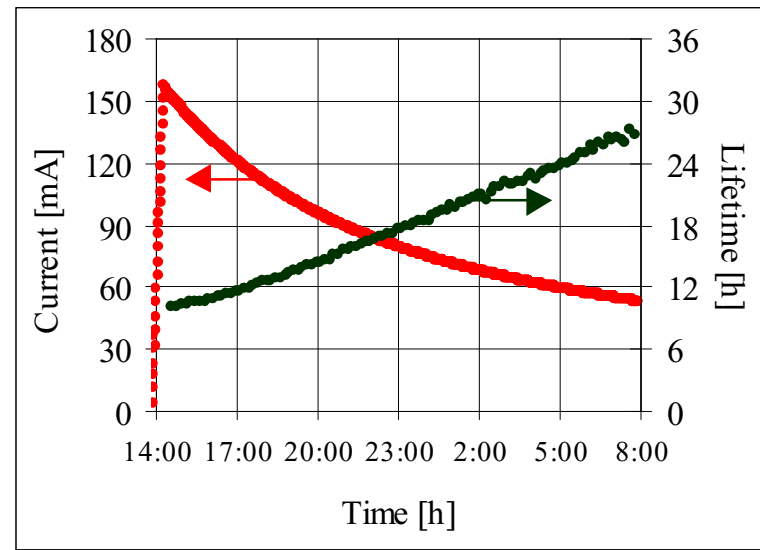

Figure 5: Stored beam intensity and lifetime versus time. Data from May $22^{\text {nd }}$ to $23^{\text {rd }} 2001$

\section{CONCLUSIONS}

One and a half year after starting the commissioning of ANKA we are running routinely to deliver synchrotron light for the commissioning of the beam lines. In a typical run we deliver a beam current of $150-180 \mathrm{~mA}$ at $2.5 \mathrm{GeV}$ two times per day.

\section{ACKNOWLEDGEMENTS}

We want to thank the staff at ANKA and at FZK that have made the commissioning possible. Especial thanks to R.Simon for performing the beam size measurement and to M.Svandrlik for helping with the cavities regulation.

\section{REFERENCES}

[1] L.Praestegaard et al., EPAC 2000, Commissioning of the ANKA injector

[2] D.Einfeld et al., EPAC 2000, Status of the $2.5 \mathrm{GeV}$ Light Source ANKA

[3] F.Zimmermann et at., SLAC-95-6792, 1995, A Fast Beam Ion Instability. 